\title{
Synthesis of Single-Nanometer-Sized Gold Nanoparticles in Liquid-Liquid Dispersion System by Femtosecond Laser Irradiation
}

\section{Takuya Okamoto, Takahiro Nakamura, Kenji Sakota, Tomoyuki Yatsuhashi}

\begin{tabular}{|c|l|}
\hline Citation & Langmuir, 35(37); 12123-12129 \\
\hline Published online & $2019-08-26$ \\
\hline Issue Date & $2019-09-17$ \\
\hline Type & Journal Article \\
\hline Textversion & author \\
\hline Highlights & $\begin{array}{l}\text { ・フェムト秒レーザーを水油の界面を利用することで、金粒子を円形状に集合させることが可能に } \\
\text { ・今後、ナノ粒子の配列・集積化による様々な用途に期待 }\end{array}$ \\
\hline Supporting & $\begin{array}{l}\text { The Supporting Information is available free of charge on the ACS Publications } \\
\text { website at https://doi.org/10.1021/acs.langmuir.9b01854. }\end{array}$ \\
\hline Rnformation & $\begin{array}{l}\text { This document is the Accepted Manuscript version of a Published Work that } \\
\text { appeared in final form in Langmuir, copyright } \text { C American Chemical Society after } \\
\text { peer review and technical editing by the publisher. To access the final edited and } \\
\text { published work see https://doi.org/10.1021/acs.langmuir.9b01854. }\end{array}$ \\
\hline DOI & \begin{tabular}{l} 
10.1021/acs.langmuir.9b01854 \\
\hline
\end{tabular} \\
\hline
\end{tabular}

\author{
Self-Archiving by Author(s) \\ Placed on: Osaka City University
}

OKAMOTO T, NAKAMURA T, SAKOTA K, \& YATSUHASHI T. (2019). Synthesis of

Single-Nanometer-Sized Gold Nanoparticles in Liquid-Liquid Dispersion System by Femtosecond Laser Irradiation. Langmuir. 35(37). 12123-12129. doi: 10.1021/acs.langmuir.9b01854 


\begin{tabular}{|c|c|}
\hline Description & 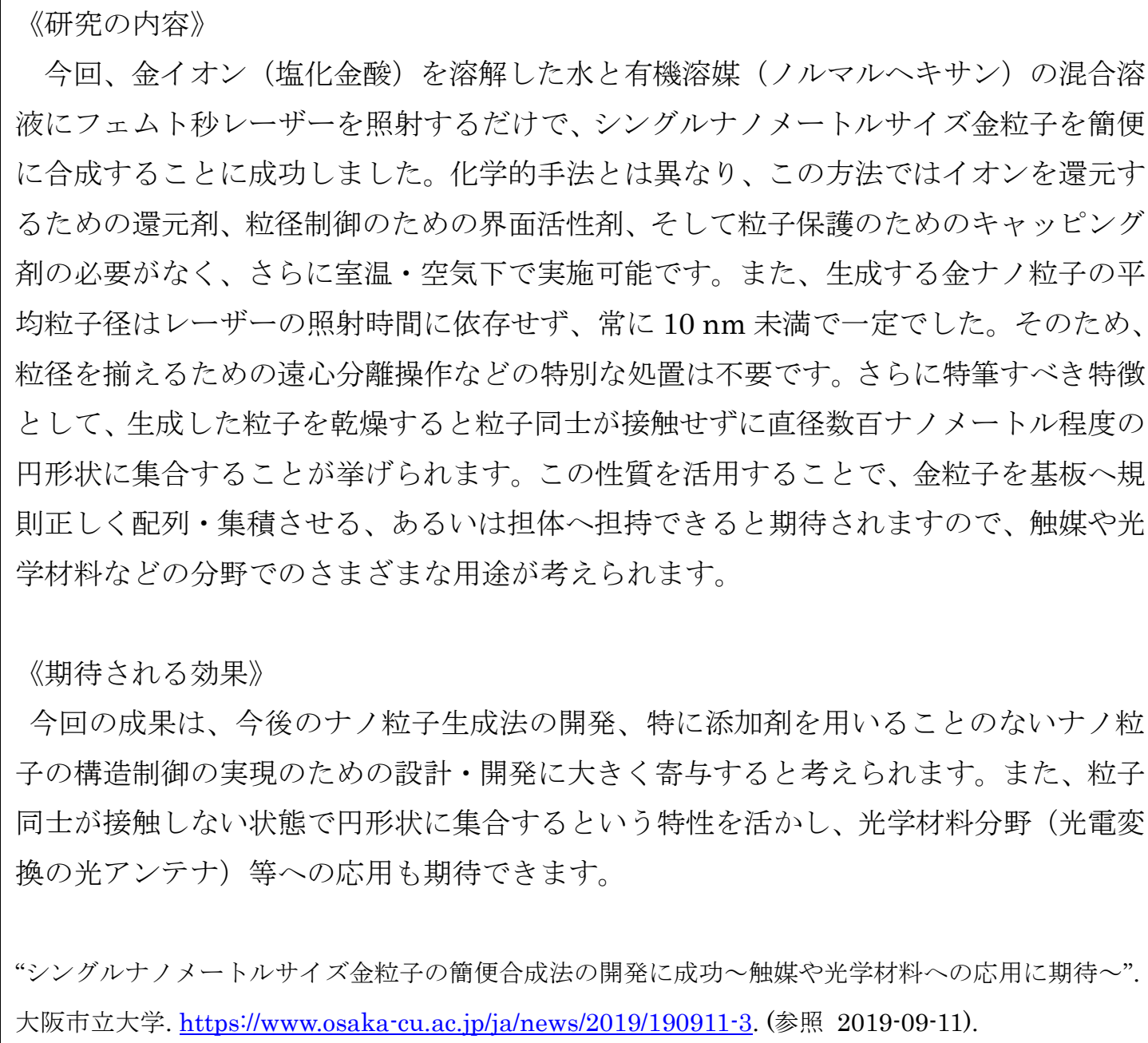 \\
\hline
\end{tabular}




\section{Synthesis of Single-Nanometer-Sized Gold}

\section{Nanoparticles in Liquid-Liquid Dispersion System by Femtosecond Laser Irradiation}

Takuya Okamoto, ${ }^{a}$ Takahiro Nakamura, ${ }^{b}$ Kenji Sakota ${ }^{a}$ Tomoyuki Yatsuhashi ${ }^{a}$ *

${ }^{\text {a }}$ Department of Chemistry, Graduate School of Science, Osaka City University, 3-3-138

Sugimoto, Sumiyoshi-ku, Osaka 558-8585 Japan

${ }^{\mathrm{b}}$ Institute of Multidisciplinary Research for Advanced Materials, Tohoku University, 2-1-1 Katahira, Aoba-ku, Sendai, Miyagi 980-8577 Japan

* To whom correspondence should be addressed. Telephone: +81-6-6605-2554. FAX: +81-66605-2552. E-mail: tomo@sci.osaka-cu.ac.jp (T.Y.)

KEYWORDS

emulsion, laser fragmentation in liquid, liquid-liquid interface, size distribution 


\section{ABSTRACT}

Gold nanoparticles (AuNPs) show unique optical properties and catalytic activities, and their synthesis from gold ions has been widely studied. One of the additive reagent-free and noncontact production procedures is the reduction of gold ions in solution by femtosecond laser pulses; however, the aggregation of AuNPs is unavoidable in homogeneous solution. Here, we report the synthesis of single-nanometer-sized AuNPs in a mixture of aqueous $\mathrm{HAuCl}_{4}$ solution and $n$-hexane (the mixture) and in aqueous $\mathrm{HAuCl}_{4}$ solution (the aqueous solution) by femtosecond laser irradiation in the absence of any additive reagents. Transmission electron microscopy revealed that circle-like colonies consisting of well-separated AuNPs were obtained from the mixture, while highly-stacked and agglomerated AuNPs were obtained from the aqueous solution. The mean size of AuNPs in the mixture was nearly independent of the laser irradiation time, whereas that obtained in aqueous solution was gradually shifted to smaller size by laser irradiation period. We propose that the adsorption of primary AuNPs on the surface of hexane microdroplets and the fragmentation of large AuNPs in water by successive laser pulses retain single-nanometer-sized AuNPs in the mixture. The use of liquidliquid interface on hexane microdroplets in aqueous solution provides a simple and useful environment to synthesize small AuNPs without the aid of surfactants or capping agents. 


\section{- INTRODUCTION}

Gold nanoparticles (AuNPs) have potential to be used as catalysts, ${ }^{1}$ medicines, ${ }^{2}$ and sensors ${ }^{3}$ due to their high surface activities and unique optical properties, such as surface plasmon resonance (SPR). Methods for the chemical synthesis of AuNPs such as the techniques of Turkevich ${ }^{4}$ and Brust-Schiffrin, ${ }^{5}$ are useful for the size-regulated and large-scale production of AuNPs. However, these methods require additive reagents such as reductants and surfactants (capping agents) in order to reduce the gold ions as well as to control the size of AuNPs. In contrast, the synthesis of nanoparticles in liquid without the aid of additive reagents has been studied by using a pulsed laser. ${ }^{6}$ Laser ablation in liquid (LAL), in which bulk materials are ablated in an inert solvent, is known to form size-controlled NPs by optimizing the laser irradiation conditions. ${ }^{6-10}$ Moreover, the strategy to produce small NPs is known as laser fragmentation in liquid (LFL), ${ }^{6-8}$ while that to produce large NPs is known as laser melting in liquid (LML). ${ }^{6,9,10}$ The energy, duration, and wavelength of laser pulses as well as the initial particle cohesion (volume, heat capacity, ionization energy, and binding energy) determine whether LFL or LML is operative. ${ }^{6}$ Another approach for forming NPs by pulsed laser irradiation is the photochemical reduction of metal ions in solution. The syntheses of not only single-element noble metal NPs $\left(\mathrm{Au},{ }^{11-19} \mathrm{Ag}^{20}\right.$ and $\left.\mathrm{Pt}^{21}\right)$ but also solidsolution alloy $\mathrm{NPs}^{22}$ in water have been reported by using femtosecond (fs) laser pulses. In particular, the synthesis of AuNPs in water by fs-laser irradiation has been well studied in terms of the photochemical reduction mechanisms of aqueous $\left[\mathrm{AuCl}_{4}\right]^{-11-14}$ and also with respect to the productivity, ${ }^{15}$ morphology,${ }^{16}$ and size distribution of AuNPs. ${ }^{17-19}$ In the abovementioned studies, reactive species such as $\mathrm{e}_{\mathrm{aq}}{ }^{-}$and $\mathrm{H}^{\bullet}$, which are generated in micro-plasma by multiphoton ionization and/or excitation of water, react with dissolved metal ions. The laser-induced plasma is formed like a filament as a result of the balance between selffocusing by a nonlinear refractive index of medium and defocusing by plasma. ${ }^{23}$ The laser 
intensity inside filaments is kept constant (ca. $10^{13} \mathrm{~W} \mathrm{~cm}^{-2}$ by intensity clamping), ${ }^{24}$ and the density of electrons in laser-induced plasma in water reaches up to $10^{18} \mathrm{~cm}^{-3} ;{ }^{25}$ however, only a small part of the solution is exposed to plasma, because the diameter of laser-induced plasma is $10-100 \mu \mathrm{m} .{ }^{24,25}$ The primary NPs formed in filaments are free to diffuse into bulk medium followed by post-reactions such as aggregation. We need to restrict aggregation to suppress the particle growth by adding surfactants or capping agents in the case of homogeneous solution. ${ }^{17,18} \mathrm{We}$ have demonstrated the use of a liquid-liquid interface between organic solvent and water for the production of characteristic nanoparticles. ${ }^{26,27}$ In this approach, reactive species are generated in water and the interface limits the diffusion of reactant from organic layer to water layer. It is also known that the liquid-liquid interface acts as a trap of solid particles. ${ }^{28}$ In this study, we report the synthesis of single-nanometer-sized AuNPs by utilizing the liquid-liquid interface of oil-in-water-type emulsions in a mixture of $n$-hexane and aqueous $\mathrm{HAuCl}_{4}$ solution. We discuss the roles of $n$-hexane microdroplets in the particle growth process based on the time evolution of morphology and size distributions of AuNPs.

\section{ם EXPERIMENTAL SECTION}

Materials. Hydrogen tetrachloroaurate (III) tetrahydrate $\left(\mathrm{HAuCl}_{4} \bullet 4 \mathrm{H}_{2} \mathrm{O}, 99 \%\right.$; Wako), distilled water (Nacalai Tesque), and $n$-hexane (spectral grade, $\geq 96 \%$; Nacalai Tesque) were used without further purification.

Laser Irradiation. We prepared an oil-in-water-type emulsion simply by mixing the aqueous $\mathrm{HAuCl}_{4}$ solution and $n$-hexane without using surfactants. $n$-Hexane was chosen because no carbon particles are formed by fs-laser irradiation. ${ }^{26}$ The mixture of the aqueous 
$\mathrm{HAuCl}_{4}$ solution and $n$-hexane was prepared by adding $1 \mathrm{~mL}$ of $n$-hexane to $1 \mathrm{~mL}$ of aqueous

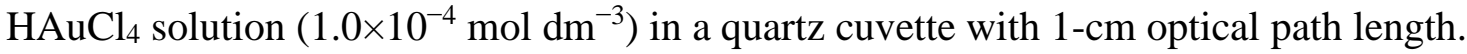
Hereinafter, the aqueous $\mathrm{HAuCl}_{4}$ solution is referred to as "the aqueous solution" and the mixture of aqueous $\mathrm{HAuCl}_{4}$ solution and $n$-hexane as "the mixture". The mixture was stirred by a Teflon-coated stir bar for $10 \mathrm{~min}$ before laser irradiation. Femtosecond laser pulses $(0.8$ $\mu \mathrm{m}, 40 \mathrm{fs}, 0.4 \mathrm{~mJ}, 1 \mathrm{kHz}$ ) were delivered from a Ti:Sapphire laser (Alpha 100/1000/XS hybrid; Thales Laser). Details of the laser experiments have been described elsewhere. ${ }^{26}$ The laser beam was focused on the central part of the cuvette by using a planoconvex lens with a focal length of $50 \mathrm{~mm}$. The mixture was stirred during the laser irradiation with a stirring speed of $3.0 \times 10^{3} \mathrm{rpm}$. The same experiments but using $1 \mathrm{~mL}$ aqueous $\mathrm{HAuCl}_{4}$ solution $\left(1.0 \times 10^{-4} \mathrm{~mol} \mathrm{dm}^{-3}\right)$ were also carried out for comparison.

Characterization of Colloidal Solutions. The UV-Vis absorption spectra were measured with a spectrophotometer (V-750; JASCO). Absorption spectrum measurements were carried out 1-min after the laser irradiation experiment. We placed a slit (height $1 \mathrm{~mm}$, width $9 \mathrm{~mm}$ ) in front of both the reference and sample optical paths of the spectrophotometer to observe only the water layer (lower layer) of the mixture. The zeta potentials of the colloidal solution in a disposable capillary cell (DTS1070; Malvern) were measured by using a dynamic light scattering instrument (Zetasizer Nano ZS; Malvern).

Characterization of Nanoparticles. The morphology and size distribution of AuNPs were observed by using a transmission electron microscope (TEM) (JEM-1010; JEOL) that was operated at an acceleration voltage of $80 \mathrm{kV}$. For the preparation of specimens for TEM observations, $10 \mu \mathrm{L}$ of solution was directly sampled onto a copper grid covered with amorphous carbon film (Nisshin EM). The excess solution was absorbed by a piece of filter paper followed by drying under air at room temperature. The mean size and size distribution 
of spherical particles were obtained by using an image processing software package (ImageJ $1.48 \mathrm{v}$ ) provided by the National Institutes of Health.

\section{- RESULTS}

Laser irradiation. A liquid-liquid dispersion system consisting of an oil-in-water-type emulsion prepared by stirring aqueous $\mathrm{HAuCl}_{4}$ solution and $n$-hexane (the mixture; Figure 1a) was exposed to femtosecond laser pulses. The mean size of $n$-hexane microdroplets in water observed using an optical microscope was about $3 \mu \mathrm{m}$ (Figure S1). The violet-colored light emitted from laser-induced plasma was observed during laser irradiation (Figure 1b). The appearance of cavitation bubbles, which are observed under static condition, ${ }^{29}$ was not identified due to the strong agitation by a stir bar. After the 10-min laser irradiation, the color of the water layer changed to pale pink, while the $n$-hexane layer remained uncolored (Figure 1c). We observed a similar emission and coloration after the aqueous $\mathrm{HAuCl}_{4}$ solution (the aqueous solution) was exposed to femtosecond laser pulses for $10 \mathrm{~min}$ (Figure 1d). The absorption spectral changes of the water layer of the mixture and the aqueous solution by 0 , 1, 2, and 10 min laser irradiation are shown in Figure 2. Before the laser irradiation, a sharp peak corresponding to the ligand-to-metal charge transfer (LMCT) band of $\mathrm{HAuCl}_{4}$ was clearly observed at $215 \mathrm{~nm}$. This LMCT peak disappeared by the 1-2 min laser irradiation, and a new peak, which was assigned to the SPR peak of AuNPs, appeared at $515 \mathrm{~nm}$. It is known that the position of the SPR peak reflects the size, morphology, and degree of aggregation of AuNPs. ${ }^{30}$ The characteristic SPR peaks of AuNPs larger than $100 \mathrm{~nm}$ in diameter, nanotriangles, and nanorods are expected to appear at $600-800 \mathrm{~nm} \cdot{ }^{31} \mathrm{We}$ conclude that these species are absent in both the mixture and the aqueous solution since the corresponding SPR peaks were not observed. It is mentioned that the wavelength of SPR 
peak taken after the 1-min (2-min) laser irradiation was longer than that taken after the 10min laser irradiation. However, there is a concern that SPR peak measured 1-min after the laser irradiation does not reflect the actual status of AuNPs just after the laser irradiation. Since the reduction of $\mathrm{Au}$ ion is not completed by the 1-min (2-min) laser irradiation, autocatalytic reduction of Au ions followed by the growth of AuNPs presumably occurs without laser irradiation. We confirmed that the monotonic absorbance increase in the UV and visible region (Figure 2a) is attributed to the Mie scattering by $n$-hexane droplets in water by separate experiments using only the mixture of water and $n$-hexane. No SPR peaks were observed in the $n$-hexane layer of the mixture after laser irradiation (Figure S2). The zeta potentials measured after the 10-min laser irradiation of the mixture and the aqueous solution were -54.5 and $-20.3 \mathrm{mV}$, respectively.

(a)

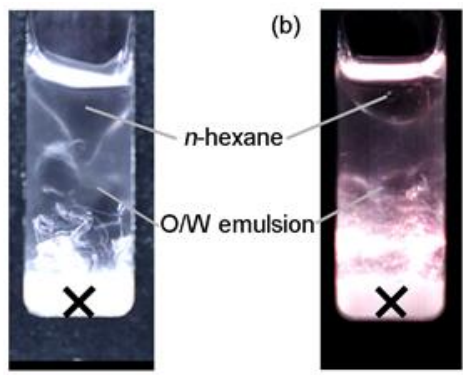

(c)

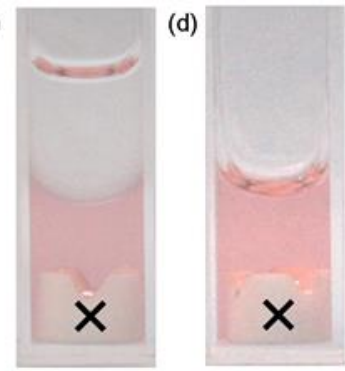

Figure 1. The appearance of the mixture of aqueous $\mathrm{HAuCl}_{4}$ solution and $n$-hexane stirred by a magnetic stirrer (a) without and (b) with laser irradiation. The appearance of (c) the mixture of aqueous $\mathrm{HAuCl}_{4}$ solution and $n$-hexane, and (d) aqueous $\mathrm{HAuCl}_{4}$ solution after the 10 -min laser irradiation. The $\times$ indicates a stir bar. 

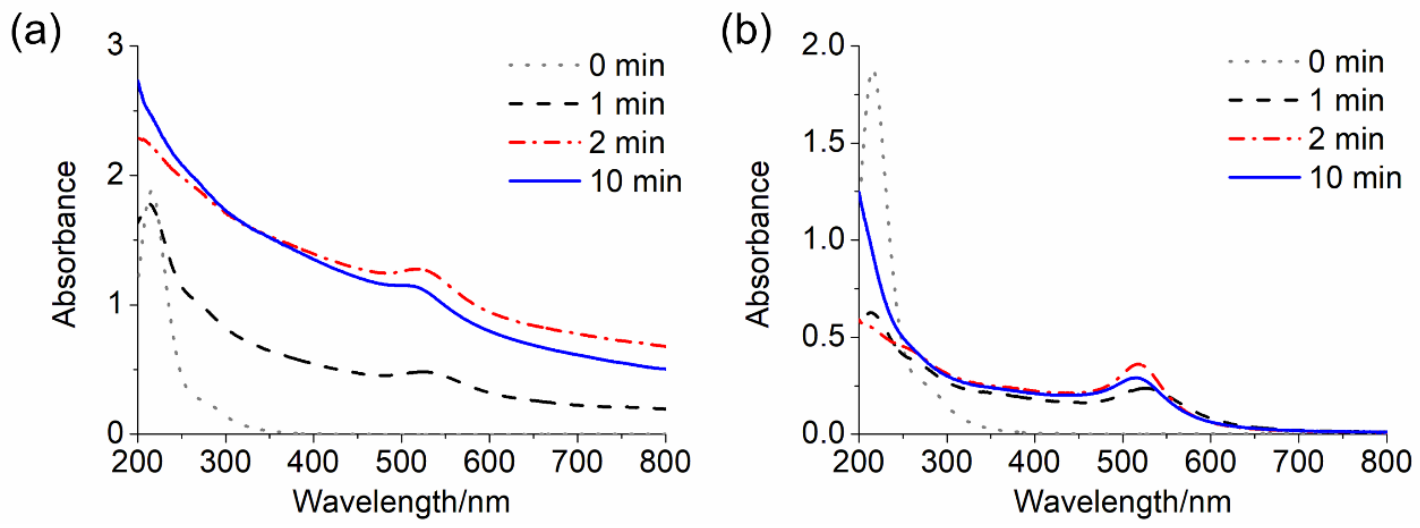

Figure 2. Absorption spectral changes of (a) the water layer of the mixture of aqueous $\mathrm{HAuCl}_{4}$ solution and $n$-hexane, and (b) aqueous $\mathrm{HAuCl}_{4}$ solution by laser irradiation.

The time evolutions of AuNPs. Figure 3 shows the TEM images and size distributions of AuNPs collected from the water layer of the mixture, and the aqueous solution after the 10-min laser irradiation. The TEM images clearly show that the AuNPs obtained in the mixture were well separated, while those obtained in the aqueous solution were highly agglomerated and stacked. Moreover, we observed circle-like colonies of AuNP monolayers in the case of the mixture (Figure 3c). The size distribution of AuNPs was bimodal for both the mixture and the aqueous solution. Here, we denote the AuNPs smaller than $10 \mathrm{~nm}$ and the AuNPs equal to or larger than $10 \mathrm{~nm}$ as $\mathrm{P} 1$ and $\mathrm{P} 2$, respectively. It is clear that P1 was dominant in the case of the mixture (Figure 3a), while P2 was the major component in the case of the aqueous solution (Figure 3b). The mean sizes of P1 and P2 in the mixture obtained by the 10-min laser irradiation were $3.4 \pm 0.9 \mathrm{~nm}$ and $14.5 \pm 3.6 \mathrm{~nm}$, respectively. Those obtained by the 10-min laser irradiation of the aqueous solution were $4.8 \pm 1.2 \mathrm{~nm}$ and $13.6 \pm 3.0 \mathrm{~nm}$, respectively. 
(a)
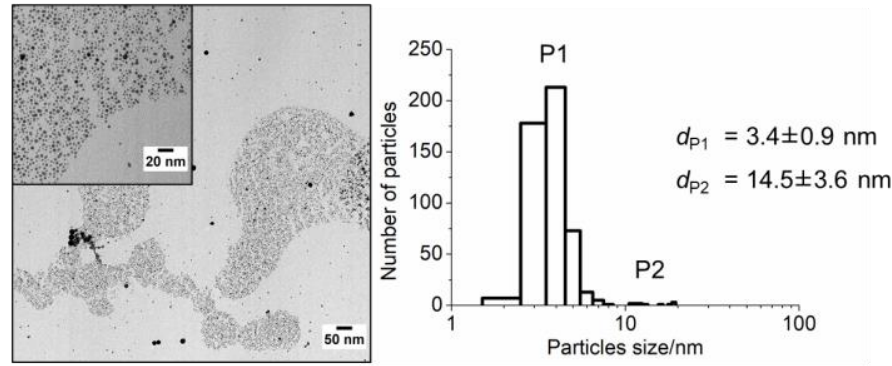

(b)
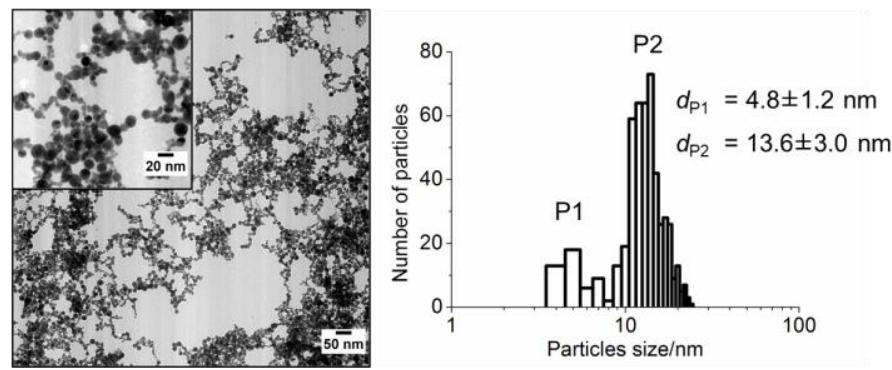

(c)
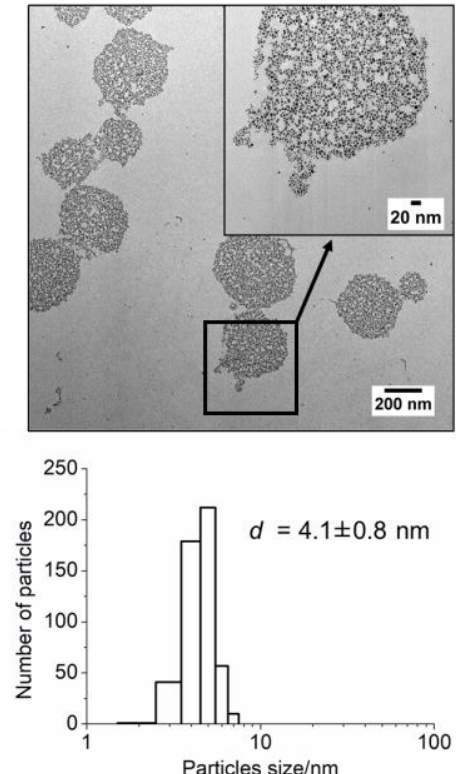

Figure 3. TEM images and size distributions of AuNPs synthesized by the 10-min fs-laser irradiation (scale bar: $50 \mathrm{~nm}$; inset, $20 \mathrm{~nm}$ ). (a) AuNPs collected from the water layer of the mixture of the aqueous $\mathrm{HAuCl}_{4}$ solution and $n$-hexane. (b) AuNPs collected from the aqueous $\mathrm{HAuCl}_{4}$ solution. $\mathrm{P} 1$ and $\mathrm{P} 2$ represent the components of bimodal distribution. $d_{\mathrm{P} 1}$ and $d_{\mathrm{P} 2}$ denote the mean size of P1 and P2, respectively. (c) TEM image of AuNPs collected from the water layer of the mixture of the aqueous $\mathrm{HAuCl}_{4}$ solution and $n$-hexane (scale bar: $200 \mathrm{~nm}$; inset, $20 \mathrm{~nm}$ ). The number of counted particles was 500 for each data point.

Figure 4 shows the time evolutions of the size distributions in the case of the mixture (Figure 4a) and the aqueous solution (Figure 4b). These size distributions were obtained from the TEM images shown in Figures S3 and S4. Although there were some fluctuations, the peak of P1 did not shift by prolonged laser irradiation in the case of AuNPs produced in the mixture. Though the number of P2 was negligible in the case of the mixture, we note that the P2 peak shifted slightly to the smaller side by prolonged laser irradiation (Figure S5). In contrast, the size distribution of AuNPs was strongly dependent on the laser irradiation time 
in the case of the aqueous solution. The mean size of AuNPs shifted from $12.7 \mathrm{~nm}(5 \mathrm{~min})$ to $6.1 \mathrm{~nm}$ (30 min). We could say that P2 gradually changed to P1 by prolonged laser irradiation in both the mixture and the aqueous solution. Figure 5a shows the time evolution of the mean size of all particles. In order to ensure the reproducibility of the experiments, we measured the size distributions of three different samples, which were obtained on three different days, for each laser irradiation time. The mean size was nearly independent of the laser irradiation time in the case of the mixture, while it gradually decreased with increasing laser irradiation time in the case of the aqueous solution. Figure $5 \mathrm{~b}$ compares the ratio of $\mathrm{P} 2$ among the total particles as a function of laser irradiation time. In the case of the mixture, P2 was $6.2 \%$ (5 min) and was decreased to $1.1 \%$ (25 min). In contrast, the P2 component was strongly dependent on the laser irradiation time in the case of the aqueous solution. P2, which was about $80 \%$ by the 5 -min laser irradiation, gradually decreased and suddenly dropped to less than $20 \%$ by the 25 -min laser irradiation. The sudden change indicated that the transformation of P2 to P1 was almost completed. However, it should be mentioned that this sudden drop was partly intrinsic to the definitions of P1 and P2: P1 and P2 are simply defined by whether the particle size was smaller than $10 \mathrm{~nm}$.

(a)

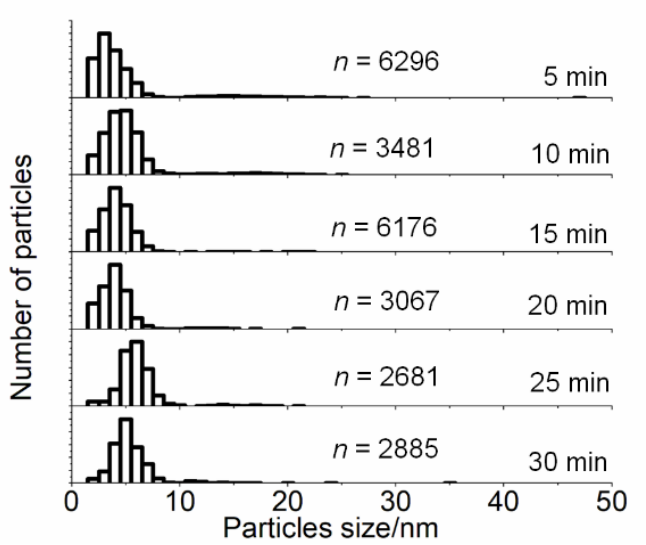

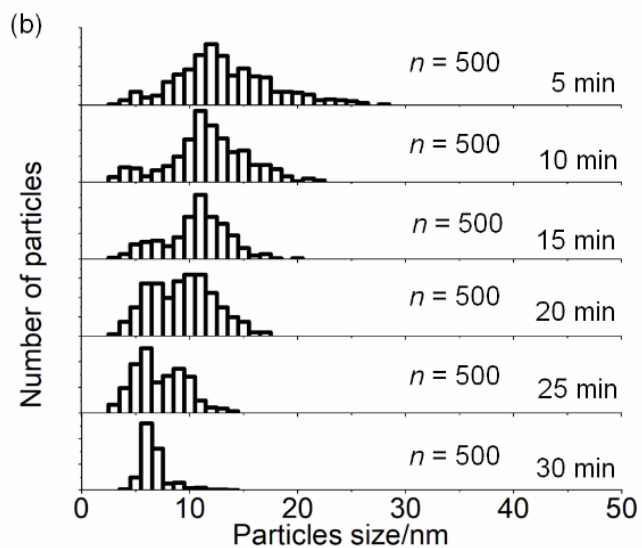

Figure 4. The time evolutions of the size distributions of AuNPs collected from (a) the water layer of the mixture of aqueous $\mathrm{HAuCl}_{4}$ and $n$-hexane, and (b) from the aqueous $\mathrm{HAuCl}_{4}$ solution. 
(a)

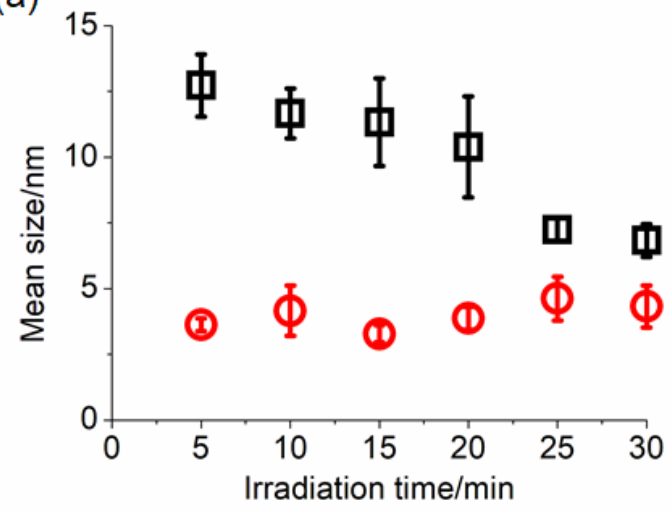

(b)

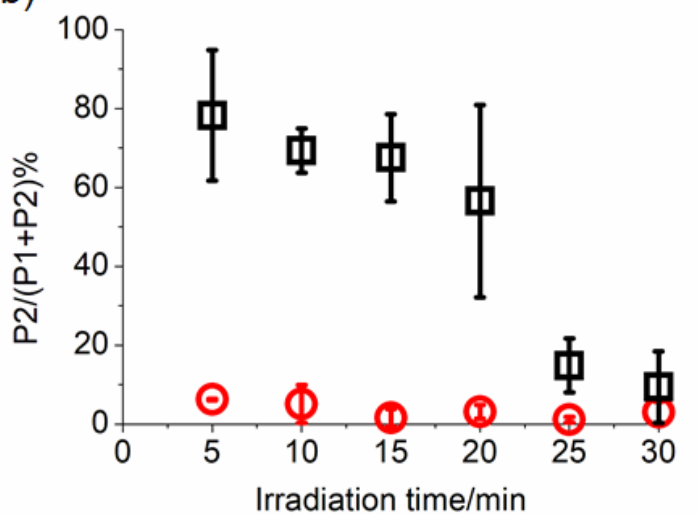

Figure 5. The time evolution of (a) the mean size and (b) the ratio of P2 among the total particles. The red circles and black squares show the results of the mixture of aqueous $\mathrm{HAuCl}_{4}$ solution and $n$-hexane, and the aqueous $\mathrm{HAuCl}_{4}$ solution, respectively. The vertical bars give the standard deviation obtained for three different samples obtained on three different days.

\section{- DISCUSSION}

The production of single-nanometer-sized AuNPs was independent of the laser irradiation time in the mixtures of aqueous $\mathrm{HAuCl}_{4}$ solution and $n$-hexane, while it required prolonged laser irradiation in the case of aqueous $\mathrm{HAuCl}_{4}$ solution. It is known that the production of single-nanometer-sized AuNPs in an aqueous $\mathrm{HAuCl}_{4}$ solution requires capping agents such as polyvinylpyrrolidone, ${ }^{17}$ and polyethylene glycol. ${ }^{18}$ In the absence of such agents, agglomerated AuNPs are obtained in aqueous $\mathrm{HAuCl}_{4}$ solution by femtosecond laser pulses. ${ }^{13-19}$ Next, therefore, we need to consider why neither capping agents nor longterm laser irradiation is required when using the mixture.

We expect that the reduction of $\mathrm{Au}$ ions to $\mathrm{Au}$ atoms followed by the nucleation of AuNPs in aqueous $\mathrm{HAuCl}_{4}$ solution ${ }^{11-14}$ is also operative in the mixture. Initially, the 
multiphoton ionization of water generates reactive species such as $\mathrm{e}^{-}$aq, $\mathrm{H}^{\bullet}$, and $\mathrm{OH}^{\bullet}$ via cationic (eq. 1) or excited states (eq. 2) of water. ${ }^{32}$

$\mathrm{H}_{2} \mathrm{O}+n h v \rightarrow \mathrm{e}^{-}+\mathrm{H}^{+}+\mathrm{OH}^{\cdot}$

$\mathrm{H}_{2} \mathrm{O}+n h v \rightarrow \mathrm{H}^{\bullet}+\mathrm{OH}^{\bullet}$

The photoelectron is immediately solvated to form hydrated electron $\mathrm{e}^{-}$aq. We can expect the reduction of $\left[\mathrm{AuCl}_{4}\right]^{-}$to neutral $\mathrm{Au}$ atoms by both $\mathrm{e}_{\mathrm{aq}}{ }^{-}$and $\mathrm{H}^{\cdot}$ (eqs. 3 and 4 ). If a sufficient amount of $\mathrm{Au}$ atoms is present, the Au atoms will aggregate to form AuNPs. On the other hand, $\mathrm{OH}^{*}$ formed by both the ionization and excitation of water acts as an oxidant. There might be the equilibrium between the reduction of $\mathrm{Au}$ ions by $\mathrm{e}_{\mathrm{aq}}{ }^{-}\left(\mathrm{H}^{\circ}\right)$ and the oxidation of $\mathrm{Au}$ atoms by $\mathrm{OH}^{*}$. However, once primary AuNPs are formed by the nucleation of Au atoms, the surface of primary AuNPs presumably be oxidized but the recovery of Au ions will not occur. It is known that $\mathrm{OH}^{\cdot}$ recombines to form $\mathrm{H}_{2} \mathrm{O}_{2}$ that catalyzes the reduction of $\mathrm{Au}$ ions in the presence of AuNPs (eq. 5). ${ }^{16}$ This autocatalytic reduction process would contribute to the increase in diameter of AuNPs that shifts SPR peak to longer wavelength without laser irradiation as observed in Figure 2.

$\left[\mathrm{AuCl}_{4}\right]^{-}+3 \mathrm{e}_{\text {aq }}^{-} \rightarrow \mathrm{Au}+4 \mathrm{Cl}^{-}$

$\left[\mathrm{AuCl}_{4}\right]^{-}+3 \mathrm{H}^{\cdot} \rightarrow \mathrm{Au}+4 \mathrm{Cl}^{-}+3 \mathrm{H}^{+}$

$\left[\mathrm{AuCl}_{4}\right]^{-}+3 / 2 \mathrm{H}_{2} \mathrm{O}_{2}+\mathrm{Au}_{m} \rightarrow \mathrm{Au}_{m+1}+3 / 2 \mathrm{O}_{2}+3 \mathrm{HCl}+\mathrm{Cl}^{-}$

Multiphoton ionization or excitation of $n$-hexane also produces $\mathrm{e}^{-}$and $\mathrm{H}^{\cdot}{ }^{33}$ The absorption spectrum of the $n$-hexane layer of the mixture after the 10-min laser irradiation (Figure S2) indicates that small amounts of byproducts are produced, as observed in $n$-hexane exposed to femtosecond pulses. ${ }^{34}$ Although it is clear that the photoreaction of $n$-hexane in the mixture 
occurs, the contribution of reactive species generated in hexane to the reduction of Au ions might be negligible, since the saturated concentration of $n$-hexane in water is $1.1 \times 10^{-4} \mathrm{~mol}$ $\mathrm{dm}^{-3}$ based on its solubility $\left(9.5 \mathrm{mg} \mathrm{dm}^{-3}, 298 \mathrm{~K}\right) .{ }^{35}$ More importantly, the multiphoton absorption cross section decreases on the order of about $10^{32}$ as the order of the multiphoton process increases. ${ }^{36}$ Therefore, multiphoton ionization of water might precede that of hexane, since the ionization potential of liquid water ${ }^{37}$ is lower than that of liquid hexane by at least $1.5 \mathrm{eV}(0.8 \mu \mathrm{m}){ }^{38}$

Although the P2 ( $\geq 10 \mathrm{~nm})$ component was dominantly formed by short-term (e.g., 5 min) laser irradiation of the aqueous solution, $\mathrm{P} 2$ was transformed to the $\mathrm{P} 1(<10 \mathrm{~nm})$ component by prolonged laser irradiation. This change is explained by LFL and in good agreement with the previous results. ${ }^{19}$ In contrast, P1 was the major component in the mixture even by short-term laser irradiation. Therefore, the P1 component in the mixture should not originate from the LFL process. In any case, the generation of the primary AuNPs in the mixture proceeds by a mechanism similar to that in the aqueous solution: the reduction of $\mathrm{Au}$ ions followed by nucleation. We propose that the mechanisms of particle growth and aggregation in the mixture are different from those in the aqueous solution. In the case of the aqueous solution, primary AuNPs grow by the aggregation with Au atoms and/or primary AuNPs from any direction (Figure 6a). When the particle growth is finished, i.e., when the Au atoms are consumed completely, the particle size is reduced via the LFL process by further laser irradiation (Figure 6b). The bimodal particle size distribution depends on the balance between the large particles grown by aggregation and the autocatalytic reaction of $\mathrm{H}_{2} \mathrm{O}_{2}$, and the small particles formed by LFL. AuNPs produced in the aqueous solution were agglomerated, and most particles were stacked after drying on a TEM grid (Figure 6c, d). 


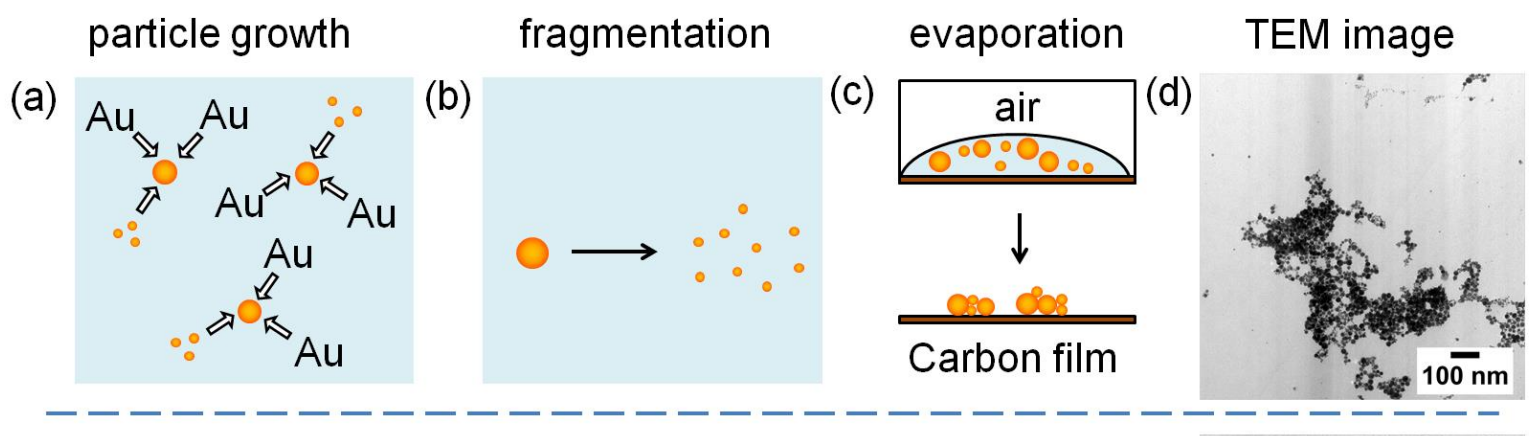

(e)

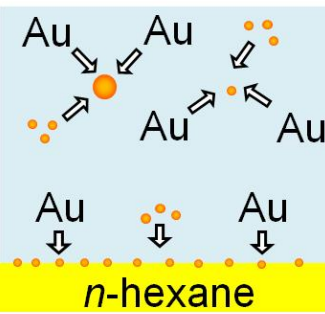

(f)

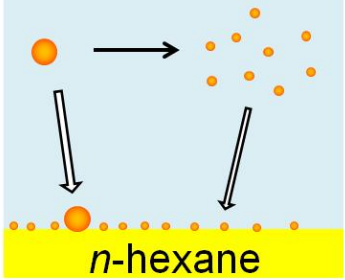

(g)

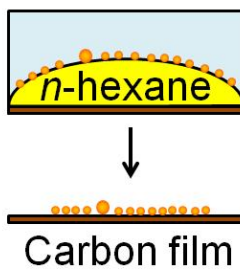

(h)

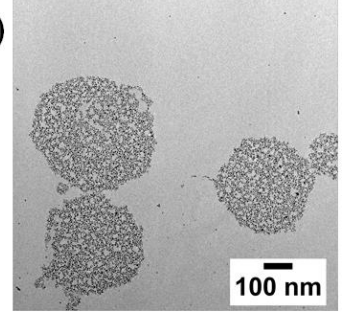

Figure 6. Schematic of the proposed mechanism of particle growth, fragmentation, and drying process on a TEM grid with a typical TEM image. $\left(\mathrm{a}^{-} \mathrm{d}\right)$ the aqueous $\mathrm{HAuCl}_{4}$ solution and $\left(\mathrm{e}^{-}\right.$ h) the mixture of the aqueous $\mathrm{HAuCl}_{4}$ solution and $n$-hexane.

Although we cannot know the exact location where AuNPs grow in the mixture, we could propose that primary AuNPs are adsorbed on the surface of $n$-hexane microdroplets based on the TEM observations. By analogy to the case of the aqueous solution, agglomeration of AuNPs in the mixture should occur during the drying process in the TEM sample preparation if AuNPs are present in bulk water. However, the AuNPs in the mixture form a circle-like colony, and each AuNP in a colony is clearly separated (Figure 3c). Therefore, we attribute the origin of the specific features of AuNPs observed in the TEM images of the mixture to the different location of AuNPs before the drying process. It is known that the neutral nanoparticles trapped on a liquid-liquid interface (e.g., hexane/water) ${ }^{39}$ agglomerate, and thus they fuse to form nanoplates (nanosheets), ${ }^{40}$ which are plate crystals with a thickness of several $\mathrm{nm}$. Charged nanoparticles trapped on an air/water ${ }^{41}$ or air/toluene ${ }^{42}$ interface form a close-packed monolayer consisting of many nanoparticles during the drying process. In addition, charged nanoparticles with ligands (e.g., dodecane 
thiol) trapped on a liquid-liquid interface (e.g., toluene/water) also form a close-packed monolayer. ${ }^{43}$ On the basis of the above-described findings, ${ }^{39-43}$ we conclude that the monolayers of AuNPs observed in the TEM images of the mixture (Figure 3c) are originated from the AuNPs trapped on the surface of $n$-hexane microdroplets (Figure 6e). The aggregation of primary AuNPs on the droplet surface might not occur due to electrostatic repulsions. In other words, single-nanometer-sized AuNPs are retained on the surface of $n$ hexane microdroplets due to the suppression of the growth of primary AuNPs. To clarify this hypothesis, it will be necessary to obtain the structural information by small-angle X-ray scattering and X-ray photon correlation spectroscopy ${ }^{44}$ or cryo-scanning electron microscopy. ${ }^{45}$

If the primary AuNPs were adsorbed on the surface of $n$-hexane microdroplets or grew in bulk water (Figure 6e), they would be P1 and P2 components, respectively. The attack of Au atoms to the primary AuNPs adsorbed on the surface of hexane droplets is limited, because no Au species ( $\mathrm{HAuCl}_{4}, \mathrm{Au}$ atom, and AuNPs) are present in $n$-hexane. In addition, the Brownian motion of AuNPs trapped on the surface is restricted to twodimensions. ${ }^{44}$ Therefore, the frequency of collision between Au atoms and the primary AuNPs adsorbed on the surface is lower than that in water. It is noted that a small amount of the P2 component observed in the mixture was transformed to P1 by LFL (Figure $6 \mathrm{f}$ ). Consequently, the monolayers of AuNPs adsorbed on the surface of hexane droplets formed a circle-like colony by drying (Figure $6 \mathrm{~g}, \mathrm{~h}$ ).

- CONCLUSIONS 
Single-nanometer-sized gold nanoparticles were dominantly synthesized in a mixture of aqueous $\mathrm{HAuCl}_{4}$ solution and $n$-hexane by femtosecond laser irradiation. Focusing femtosecond laser pulses into water produced reactive species that reduced Au ions to neutral $\mathrm{Au}$ atoms, and then aggregated them to form AuNPs. In contrast, when the mixture was used rather than the aqueous solution, the adsorption of primary AuNPs on the surface of hexane microdroplets suppressed the particle growth process due to the low probability of aggregation. In addition, LFL might have contributed to the limitation of AuNP size. Of course, the present method could be improved in several ways. First, although we reduced the ratio of large particles ( $\geq 10 \mathrm{~nm}$ ) down to a $1.1 \%$ among the total particles, we could not completely eradicate the large particles. Second, the distribution width of single-nanometersized gold NPs is similar to that obtained in water by femtosecond laser pulse irradiation in the presence of capping agents, ${ }^{17,18}$ but still wider than that obtained by the chemical reduction method with capping agents. ${ }^{46}$ Sachdev et al. reported that the size and shape of the AuNPs produced by chemical synthesis method in an oil-in-water-type emulsion are dependent not only on the concentration of reactants but also on the size of oil-droplets. ${ }^{39}$ To address these issues, we need to optimize the emulsion conditions, including the size and number of the droplets, by using other organic solvents, and by optimizing the temperature and stirring speed etc.

\section{ASSOCIATED CONTENT}

\section{Supporting Information.}

The Supporting Information is available free of charge on the ACS Publications website at DOI: 10.1021/acs.langmuir.xxxx. 
Optical microscope image of $n$-hexane droplets in water before and after the 10 -min laser irradiation, absorption spectrum of the hexane layer of the mixture exposed to femtosecond laser pulses for $10 \mathrm{~min}$, TEM images of AuNPs collected from the water layer of the mixture or the aqueous solution exposed to femtosecond laser pulses for 5, 10, 15, 20, 25 and 30-min, and time evolution of size distributions of P2 component in the mixture.

\section{AUTHOR INFORMATION}

\section{Corresponding Author}

Telephone: +81-6-6605-2554. FAX: +81-6-6605-2552. E-mail: tomo@ sci.osaka-cu.ac.jp (T.Y.).

\section{Author Contributions}

The manuscript was written through contributions of all authors. All authors have given approval to the final version of the manuscript.

\section{Notes}

The authors declare no competing financial interest.

\section{ACKNOWLEDGMENT}

The present research was supported in part by JSPS KAKENHI Grant Number $18 \mathrm{~J} 15442$ for JSPS Research Fellow. This work was performed under the Research Program for Next Generation Young Scientists of "Five-star Alliance" in "NJRC Mater. \& Dev" for T. O. We thank Mr. Yuhei Tahara and Prof. Makoto Miyata for their help with TEM experiments and observation of microdroplets. We thank Prof. Satoshi Shinoda for his help with zeta potential 
measurements. We thank Mr. Kazuhiko Kondo of Thales Japan Inc. for his kind contribution to our laser system.

\section{ABBREVIATIONS}

AuNPs, gold nanoparticles; fs, femtosecond; LAL, laser ablation in liquid; LFL, laser fragmentation in liquid; LMCT, ligand-to-metal charge transfer; LML, laser melting in liquid; SPR, surface plasmon resonance; TEM, transmission electron microscope.

\section{REFERENCES}

(1) Haruta, M.; Kobayashi, T.; Sano, H.; Yamada, N. Novel Gold Catalysts for the Oxidation of Carbon Monoxide at a Temperature Far Below $0{ }^{\circ} \mathrm{C}$. Chem. Lett. 1987, 16 (2), 405408.

(2) Boisselier, E.; Astruc, D. Gold Nanoparticles in Nanomedicine: Preparations, Imaging, Diagnostics, Therapies and Toxicity. Chem. Soc. Rev. 2009, 38 (6), 1759-1782.

(3) Saha, K.; Agasti, S. S.; Kim, C.; Li, X.; Rotello, V. M. Gold Nanoparticles in Chemical and Biological Sensing. Chem. Rev. 2012, 112 (5), 2739-2779.

(4) Turkevich, J.; Stevenson, P. C.; Hillier, J. A Study of the Nucleation and Growth Processes in the Synthesis of Colloidal Gold. Discuss. Faraday Soc. 1951, 11, 55-75.

(5) Brust, M.; Walker, M.; Bethell, D.; Schiffrin, D. J.; Whyman, R. Synthesis of ThiolDerivatised Gold Nanoparticles in a Two-Phase Liquid-Liquid System. J. Chem. Soc. Chem. Commun. 1994, 801-802. 
(6) Zhang, D.; Gökce, B.; Barcikowski, S. Laser Synthesis and Processing of Colloids: Fundamentals and Applications. Chem. Rev. 2017, 117 (5), 3990-4103.

(7) Werner, D.; Furube, A.; Okamoto, T.; Hashimoto, S. Femtosecond Laser-Induced Size Reduction of Aqueous Gold Nanoparticles: In Situ and Pump-Probe Spectroscopy Investigations Revealing Coulomb Explosion. J. Phys. Chem. C 2011, 115 (17), 85038512.

(8) Delfour, L.; Itina, T. E. Mechanisms of Ultrashort Laser-Induced Fragmentation of Metal Nanoparticles in Liquids: Numerical Insights. J. Phys. Chem. C 2015, 119 (24), $13893-$ 13900.

(9) Inasawa, S.; Sugiyama, M.; Yamaguchi, Y. Laser-Induced Shape Transformation of Gold Nanoparticles below the Melting Point: The Effect of Surface Melting. J. Phys. Chem. B 2005, 109 (8), 3104-3111.

(10) Link, S.; Burda, C.; Mohamed, M. B.; Nikoobakht, B.; El-Sayed, M. A. Laser Photothermal Melting and Fragmentation of Gold Nanorods: Energy and Laser PulseWidth Dependence. J. Phys. Chem. A 2002, 103 (9), 1165-1170.

(11) John, M. G.; Meader, V. K.; Moore Tibbetts, K. Au Nanoparticle Synthesis via Femtosecond Laser-Induced Photochemical Reduction of $\left[\mathrm{AuCl}_{4}\right]^{-}$. In Photochemistry and Photophysics - Fundamentals to Applications; InTech, 2018.

(12) Nakashima, N.; Yamanaka, K. I.; Saeki, M.; Ohba, H.; Taniguchi, S.; Yatsuhashi, T. Metal Ion Reductions by Femtosecond Laser Pulses with Micro-Joule Energy and Their Efficiencies. J. Photochem. Photobiol. A Chem. 2016, 319-320, 70-77. 
(13) Moore Tibbetts, K.; Tangeysh, B.; Odhner, J. H.; Levis, R. J. Elucidating Strong Field Photochemical Reduction Mechanisms of Aqueous $\left[\mathrm{AuCl}_{4}\right]^{-}$: Kinetics of Multiphoton Photolysis and Radical-Mediated Reduction. J. Phys. Chem. A 2016, 120 (20), 35623569.

(14) Meader, V. K.; John, M. G.; Rodrigues, C. J.; Moore Tibbetts, K. Roles of Free Electrons and $\mathrm{H}_{2} \mathrm{O}_{2}$ in the Optical Breakdown-Induced Photochemical Reduction of Aqueous $\left[\mathrm{AuCl}_{4}\right]^{-}$. J. Phys. Chem. A 2017, 121 (36), 6742-6754.

(15) Muttaqin; Nakamura, T.; Sato, S. Synthesis of Gold Nanoparticle Colloids by Highly Intense Laser Irradiation of Aqueous Solution by Flow System. Appl. Phys. A Mater. Sci. Process. 2015, 120 (3), 881-888.

(16) Tangeysh, B.; Moore Tibbetts, K.; Odhner, J. H.; Wayland, B. B.; Levis, R. J. Gold Nanotriangle Formation through Strong-Field Laser Processing of Aqueous $\mathrm{KAuCl}_{4}$ and Postirradiation Reduction by Hydrogen Peroxide. Langmuir 2017, 33 (1), 243-252.

(17) Nakamura, T.; Mochidzuki, Y.; Sato, S. Fabrication of Gold Nanoparticles in Intense Optical Field by Femtosecond Laser Irradiation of Aqueous Solution. J. Mater. Res. 2008, 23 (4), 968-974.

(18) Tangeysh, B.; Moore Tibbetts, K.; Odhner, J. H.; Wayland, B. B.; Levis, R. J. Gold Nanoparticle Synthesis Using Spatially and Temporally Shaped Femtosecond Laser Pulses: Post-Irradiation Auto-Reduction of Aqueous $\left[\mathrm{AuCl}_{4}\right]^{-}$. J. Phys. Chem. C 2013, 117 (36), 18719-18727.

(19) Nakamura, T.; Herbani, Y.; Ursescu, D.; Banici, R.; Dabu, R. V.; Sato, S. Spectroscopic Study of Gold Nanoparticle Formation through High Intensity Laser Irradiation of Solution. AIP Adv. 2013, 3 (8), 082101. 
(20) Nakamura, T.; Magara, H.; Herbani, Y.; Sato, S. Fabrication of Silver Nanoparticles by Highly Intense Laser Irradiation of Aqueous Solution. Appl. Phys. A 2011, 104 (4), 1021-1024.

(21) Nakamura, T.; Takasaki, K.; Ito, A.; Sato, S. Fabrication of Platinum Particles by Intense, Femtosecond Laser Pulse Irradiation of Aqueous Solution. Appl. Surf. Sci. 2009, 255 (24), 9630-9633.

(22) Sarker, M. S. I.; Nakamura, T.; Sato, S. All-Proportional Solid-Solution Rh-Pd-Pt Alloy Nanoparticles by Femtosecond Laser Irradiation of Aqueous Solution with Surfactant. J. Nanoparticle Res. 2015, 17 (6), 1-14.

(23) Nakashima, N.; Yamanaka, K.; Itoh, A.; Yatsuhashi, T. Ionic Valence Change of Metal Ions in Solution by Femtosecond Laser Excitation Accompanied by White-Light Laser. Chin. J. Phys. 2014, 52 (1) 504-518.

(24) Milián, C.; Jarnac, A.; Brelet, Y.; Jukna, V.; Houard, A.; Mysyrowicz, A.; Couairon, A. Effect of Input Pulse Chirp on Nonlinear Energy Deposition and Plasma Excitation in Water. J. Opt. Soc. Am. B 2014, 31 (11), 2829-2837.

(25) Chin, S. L.; Hosseini, S. A.; Liu, W.; Luo, Q.; Théberge, F.; Aközbek, N.; Becker, A.; Kandidov, V. P.; Kosareva, O. G.; Schroeder, H. The Propagation of Powerful Femtosecond Laser Pulses in Opticalmedia: Physics, Applications, and New Challenges. Can. J. Phys. 2005, 83 (9), 863-905.

(26) Hamaguchi, T.; Okamoto, T.; Mitamura, K.; Matsukawa, K.; Yatsuhashi, T. Synthesis of Hydrophilic and Hydrophobic Carbon Nanoparticles from Benzene/Water Bilayer Solution with Femtosecond Laser Generated Plasma Filaments in Water. Bull. Chem. Soc. Jpn. 2015, 88 (2), 251-261. 
(27) Okamoto, T.; Mitamura, K.; Hamaguchi, T.; Matsukawa, K.; Yatsuhashi, T. Synthesis of Fluorine-Doped Hydrophilic Carbon Nanoparticles from Hexafluorobenzene by Femtosecond Laser Pulses. ChemPhysChem 2017, 18 (9), 1007-1011.

(28) Binks, B. P. Particles as Surfactants-Similarities and Differences. Curr. Opin. Colloid Interface Sci. 2002, 7 (1-2), 21-41.

(29) Uwada, T.; Wang, S. F.; Liu, T.-H.; Masuhara, H. Preparation and Micropatterning of Gold Nanoparticles by Femtosecond Laser-induced Optical Breakdown. J. Photochem. Photobiol. A 2018, 346, 177-186.

(30) Amendola, V.; Meneghetti, M. Size Evaluation of Gold Nanoparticles by UV-Vis Spectroscopy. J. Phys. Chem. C 2009, 113 (11), 4277-4285.

(31) Attia, Y. A.; Buceta, D.; Requejo, F. G.; Giovanetti, L. J.; López-Quintela, M. A. Photostability of Gold Nanoparticles with Different Shapes: The Role of Ag Clusters. Nanoscale 2015, 7 (26), 11273-11279.

(32) Chin, S. L.; Lagace, S. Generation of $\mathrm{H}_{2}, \mathrm{O}_{2}$, and $\mathrm{H}_{2} \mathrm{O}_{2}$ from Water by the Use of Intense Femtosecond Laser Pulses and the Possibility of Laser Sterilization. Appl. Opt. 1996, 35 (6), 907-911.

(33) Sander, M. U.; Brummund, U.; Luther, K.; Troe, J. Subpicosecond Transient Absorption Study of the UV Two-Photon Excitation of Liquid Alkanes. J. Phys. Chem. 1993, 97 (32), 8378-8383.

(34) Sato, Y.; Kodama, T.; Shiromaru, H.; Sanderson, J. H.; Fujino, T.; Wada, Y.; Wakabayashi, T.; Achiba, Y. Synthesis of Polyyne Molecules from Hexane by Irradiation of Intense Femtosecond Laser Pulses. Carbon 2010, 48 (5), 1673-1676. 
(35) McAuliffe, C. Solubility in Water of Paraffin, Cycloparaffin, Olefin, Acetylene, Cycloolefin, and Aromatic Hydrocarbons 1. J. Phys. Chem. 1966, 70 (4), 1267-1275.

(36) Yatsuhashi, T.; Ichikawa, S.; Shigematsu, Y.; Nakashima, N. High-Order Multiphoton Fluorescence of Organic Molecules in Solution by Intense Femtosecond Laser Pulses. J. Am. Chem. Soc. 2008, 130 (46), 15264-15265.

(37) Nikogosyan, D. N.; Oraevsky, A. A.; Rupasov, V. I. Two-Photon Ionization and Dissociation of Liquid Water by Powerful Laser UV Radiation. Chem. Phys. 1983, 77 (1), 131-143.

(38) Casanovas, J.; Grob, R.; Delacroix, D.; Guelfucci, J. P.; Blanc, D. Photoconductivity Studies in Some Nonpolar Liquids. J. Chem. Phys. 1981, 75 (9), 4661-4668.

(39) Sachdev, S.; Maugi, R.; Woolley, J.; Kirk, C.; Zhou, Z.; Christie, S. D. R.; Platt, M. Synthesis of Gold Nanoparticles Using the Interface of an Emulsion Droplet. Langmuir 2017, 33 (22), 5464-5472.

(40) Edel, J. B.; Kornyshev, A. A.; Kucernak, A. R.; Urbakh, M. Fundamentals and Applications of Self-Assembled Plasmonic Nanoparticles at Interfaces. Chem. Soc. Rev. 2016, 45 (6), 1581-1596.

(41) Martin, M. N.; Basham, J. I.; Chando, P.; Eah, S.-K. Charged Gold Nanoparticles in Non-Polar Solvents: 10-Min Synthesis and 2D Self-Assembly. Langmuir 2010, 26 (10), $7410-7417$.

(42) Park, Y. K.; Park, S. Directing Close-Packing of Midnanosized Gold Nanoparticles at a Water/Hexane Interface. Chem. Mater. 2008, 20 (6), 2388-2393. 
(43) Rao, C. N. R.; Kulkarni, G. U.; Thomas, P. J.; Agrawal, V. V.; Saravanan, P. Films of Metal Nanocrystals Formed at Aqueous-Organic Interfaces. J. Phys. Chem. B 2003, 107 (30), 7391-7395.

(44) Cui, M.; Miesch, C.; Kosif, I.; Nie, H.; Kim, P. Y.; Kim, H.; Emrick, T.; Russell, T. P. Transition in Dynamics as Nanoparticles Jam at the Liquid/Liquid Interface. Nano Lett. 2017, $17(11), 6855-6862$.

(45) Isa, L.; Lucas, F.; Wepf, R.; Reimhult, E. Measuring Single-Nanoparticle Wetting Properties by Freeze-Fracture Shadow-Casting Cryo-Scanning Electron Microscopy. Nat. Commun. 2011, 2, 438.

(46) Hussain, I.; Graham, S.; Wang, Z.; Tan, B.; Sherrington, D. C.; Rannard, S. P.; Cooper, A. I.; Brust, M. Size-Controlled Synthesis of Near-Monodisperse Gold Nanoparticles in the 1-4 nm Range Using Polymeric Stabilizers. J. Am. Chem. Soc. 2005, 127 (47), 16398-16399. 


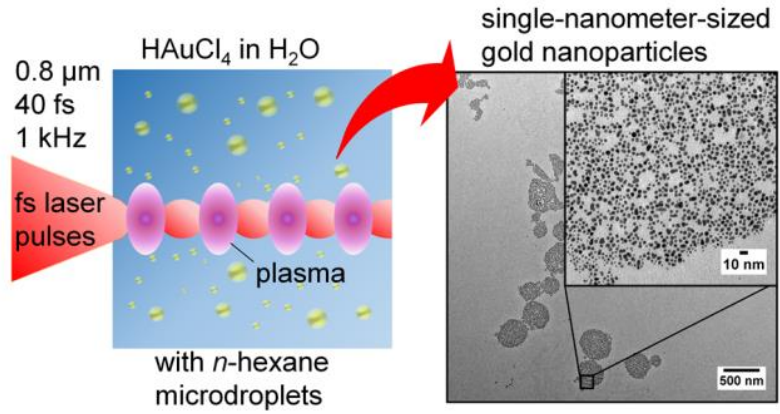

